\title{
Proof-of-concept demonstrator for the off-axis retroreflector interferometer configuration for NGGM
}

Christian Dahl, Gino Bruno Amata, Luciana Bonino, Andreas Baatzsch, Johanna Flock, et al.

Christian Dahl, Gino Bruno Amata, Luciana Bonino, Andreas Baatzsch, Johanna Flock, Arnaud Heliere, Luca Massotti, Kolja Nicklaus, Marco Pisani, Massimo Zucco, "Proof-of-concept demonstrator for the off-axis retroreflector interferometer configuration for NGGM," Proc. SPIE 11852, International Conference on Space Optics - ICSO 2020, 118523I (11 June 2021); doi: $10.1117 / 12.2599605$ SPIE. Event: International Conference on Space Optics - ICSO 2021, 2021, Online 


\section{International Conference on Space Optics-ICSO 2020}

Virtual Conference

30 March-2 April 2021

Edited by Bruno Cugny, Zoran Sodnik, and Nikos Karafolas
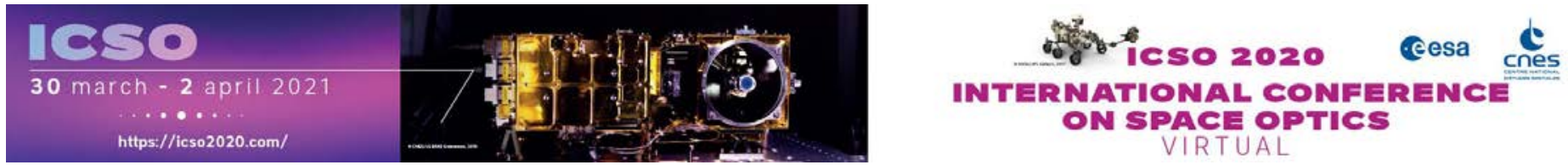

\section{Proof-of-concept demonstrator for the off-axis retroreflector interferometer configuration for NGGM}

\section{Cesa issopereatings denes}




\title{
Proof-of-Concept Demonstrator for the Off-Axis Retroreflector Interferometer Configuration for NGGM
}

\author{
Christian Dahl ${ }^{\mathrm{a}}$, Gino Bruno Amata ${ }^{\mathrm{b}}$, Luciana Bonino ${ }^{\mathrm{b}}$, Andreas Baatzsch ${ }^{\mathrm{a}}$, Johanna Flock ${ }^{\mathrm{a}}$, Arnaud \\ Heliere $^{\mathrm{d}}$, Luca Massotti ${ }^{\mathrm{d}}$, Kolja Nicklaus ${ }^{\mathrm{a}}$, Marco Pisani ${ }^{\mathrm{c}}$, Massimo Zucco ${ }^{\mathrm{c}}$

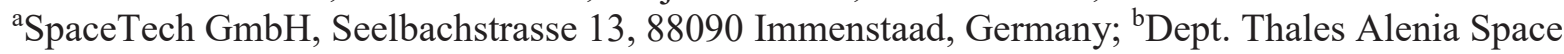 \\ Italia S.p.A, Strada Antica di Collegno 253, 10146 Torino, Italy; ' Istituto Nazionale di Ricerca \\ Metrologica, Str. delle Cacce 91, 10135 Torino, Italy; ${ }^{\mathrm{d} E u r o p e a n ~ S p a c e ~ R e s e a r c h ~ a n d ~ T e c h n o l o g y ~}$ \\ Centre, Keplerlaan 1, 2201 AZ Noordwijk, The Netherlands
}

\begin{abstract}
This paper presents the design and breadboarding of the proof of concept demonstrator for the so called retro-reflector interferometer scheme in off-axis configuration for the 'Next Generation Gravity Mission' (NGGM) studied at the European Space Agency (ESA). This configuration can offer benefits in terms of overall satellite configuration compared to the transponder scheme, which is currently flying on board of GRACE-FO. However, it relies on very low received laser signal levels due to the fact that the laser light is travelling about $100 \mathrm{~km}$ from the master satellite to the remote satellite and is reflected back to the master satellite by a retro-reflector. In comparison to the transponder scheme, where the signal is amplified on the remote satellite using a laser, which is optically phase locked to the laser signal of the master spacecraft, this reflection does not amplify the signal. Thus, even with higher emitted laser power, instead of some nanowatt, only a few picowatt are available on the according science detector. Therefore, less than a femtowatt of straylight within the detectable heterodyne frequency and angular range is allowed on the detector to fulfil the ranging noise requirement.
\end{abstract}

The paper gives insights into the main opto-mechanical design topics of the Optical Bench Assembly (OBA). It includes the optical analysis results as well as mechanical design to suppress straylight below the required limit. The optomechanical design of the OBA is complemented by the opto-mechanical design of the test setup and by the electro-optical design of the phase read-out chain. Finally, preliminary results from the test campaign are presented.

Keywords: Laser interferometer, LTI, LMI, Mass Change, NGGM, GRACE FO

\section{INTRODUCTION AND TOP LEVEL REQUIREMENTS}

The measurement of the static and temporal variation of Earth's gravity field yields important information on water storage, seasonal and sub-seasonal water cycles, their impact on water levels and delivers key data to Earth's climate models. The satellite missions GOCE (ESA), GRACE (US-DE) and just recently GRACE Follow-On (US-GER) resulted in a significant improvement on our understanding of the system Earth. ESA is investigating the concept of a 'Next Generation Gravity Mission' (NGGM) as a Mission of Opportunity for ESA-NASA cooperation in view of their Mass Change and Geoscience International Constellation (MAGIC) [9]: it consists of two pairs of satellites embarking a heterodyne laser interferometer for inter-satellite ranging, the so called Laser Tracking Instrument (LTI), to further improve the measurement accuracy of the time-variable Earth gravity field.

For the LTI two basic interferometer implementations are under investigation: the transponder scheme, similar to the successfully implemented LRI on GRACE-FO[1], and the retroreflector scheme as proposed in former ESA studies with low inter-satellite distance, found optimal for scientific applications around $100 \mathrm{~km}$. Both schemes have been presented in detail e.g. in [2] and we refer the reader to this publication for an overall understanding of the instrument concept.

In order to respond to the wide science user community, ESA aims to achieve an improved measurement of the variable Earth's gravity field, in terms of temporal and spatial resolution, to better track the mass changes within the Earth "system". The mission objectives, as stated in Phase 0, are the measurement of the geoid with $1 \mathrm{~mm}$ accuracy at 3-day intervals with 
$<500 \mathrm{~km}$ spatial resolution, and at 10-day intervals with $<150 \mathrm{~km}$ spatial resolution, over a time span of at least 7 years $[3]$.

For the LTI the top level science requirement is broken down into a top level LTI measurement noise threshold and goal requirement of

$$
\begin{gathered}
\tilde{x}_{t h r} \leq 20 \frac{\mathrm{nm}}{\sqrt{\mathrm{Hz}}} x \operatorname{NSF}(f) \quad \text { for } 1 \times 10^{-4} \mathrm{~Hz}<f<1 \mathrm{~Hz} \\
\tilde{x}_{\text {goal }} \leq 10 \frac{\mathrm{nm}}{\sqrt{\mathrm{Hz}}} \times N S F(f) \quad \text { for } 1 \times 10^{-4} \mathrm{~Hz}<f<1 \mathrm{~Hz} \\
\text { With } N S F(f)=\sqrt{1+\left(\frac{10 \mathrm{mHz}}{f}\right)^{2}}
\end{gathered}
$$

This is almost an order of magnitude more demanding than the required LRI performance on GRACE FO.

In [2] it is discussed that both interferometer schemes are considered capable to provide the required performance, but that the retroreflector scheme needs further investigation, mainly due to a challenging stray light suppression requirement on the optical bench and the potential occurrence of cycle slips. The issue of straylight suppression has been investigated in this activity.

For the optical bench OBA we have allocated an allowed ranging measurement noise of $2 \mathrm{~nm} / \sqrt{ } \mathrm{Hz} \times \mathrm{NSF}(\mathrm{f})$ [2]. The optical bench design needs to fulfil (among the other requirements detailed in [2]) the following key requirements to achieve this:

1. A low phase noise between the local oscillator laser beam (LO beam) and the transmitted beam that is sent to the remote satellite (TX beam) and ultimately received back on the master satellite as RX beam on the optical bench;

2. a sufficiently high heterodyne amplitude on the science photodetector and a low noise signal evaluation chain;

3. a relative suppression of straylight of more than 4 to 6 orders of magnitude with respect to the received science signal.

With a TX power of $500 \mathrm{~mW}$ and a RX power between 1.8 and $5.6 \mathrm{pW}$ [2] a straylight suppression of about 150 to 170 $\mathrm{dB}$ of any spurious TX light, within a cone of about $1 \mathrm{mrad}$ around the LO beam, is required. Straylight at larger angles of incidence are strongly suppressed in the heterodyne detection. While the above requirements 1 and 2 can be achieved by normal engineering and analysis work, requirement number 3 is far more difficult to analyze with sufficient accuracy: $n$ experimental verification with representative optical bench hardware is considered absolutely essential.

In section 2, we describe the optical bench design concept, the implemented design, and the required Ground Support Equipment (GSE) to perform the experimental investigation, including the utilized laser source and read-out chain. In section 3 we present and discuss the preliminarily achieved test results and draw our conclusions in section 5 .

\section{ANALYSIS AND DESIGN}

A schematic of the NGGM proof-of-concept demonstrator for the off-axis retroreflector interferometer configuration is presented in Figure 1. The OBA is shown in the center of the schematic and the most important parts of the surrounding test setup are the two retroreflectors (RR1 and RR2) and the attenuator (ATT2). The GSE for the laser source is not shown in this figure but will be explained in more detail in the section "laser source GSE".

The opto-mechanical design of the OBA is very close to a possible FM of the instrument and it is mainly based on design rules and hardware of the LRI flying on GRACE-FO. The fiber collimator 1 (Fiber 1), through which the beam that is send to the other spacecraft is delivered (TX beam), is mounted at the outer surface of the OBA and the polarisation of the light from fiber collimator 1 is cleaned up using the polariser 1 (Pol1). The unwanted polarisation is directed into beam dump 1 (BD1). The implementation of the fiber collimator 2 (Fiber2), the polarisator 2 (Pol2) and beam dump 2 (BD2) is identical.

The beam splitter 1 (BS1) serves as optical power beam splitter and as combination beam splitter. First, the beam splitter splits the power of the beam coming from fiber collimator 1 into two parts. $99 \%$ is directed as TX beam towards retro 
reflector 1 (RR1), which directs the light towards retroreflector 2 (RR2) and ultimately enters the OBA as RX beam again. $1 \%$ of the light from fiber collimator 1 is directed towards attenuator 1 (ATT1) inside the OBA. $90 \%$ of the light from fiber collimator 2, through which the local oscillator beam is delivered (LO beam) is reflected at beam splitter 2 (BS2) and afterwards at BS1. At BS1 the combination of the light beams from fiber collimators 1 and 2 happens and the reference interferometer is formed. Both beams travel toward attenuator 1 (ATT1), which reflects $99 \%$ of the light towards beam dump 3 (BD3). The attenuator serves the purpose to suppress straylight on quadrant photodiode 2 from quadrant photodiode 1. After the attenuator, both beams are propagating through an aperture and are focussed with a lens on the quadrant photodiode 1 (QPD1), where the beat note between both beams is detected. The aperture reduces the straylight from quadrant photodiode 1 in addition to the attenuator 1.

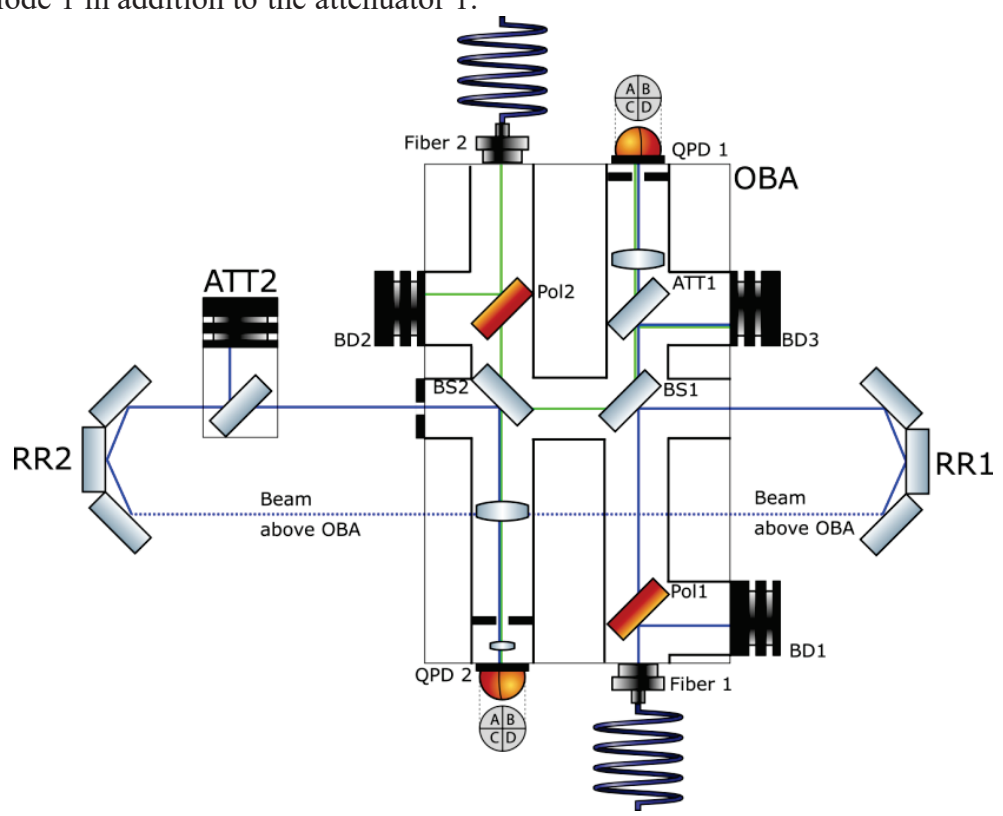

Figure 1 Schematic of the proof-of-concept demonstrator for the off-axis retroreflector interferometer configuration for NGGM.

The beam splitter 2 (BS2) serves as power splitter and as combination beam splitter, too. First, the beam splitter splits the power of the beam coming from fiber collimator 2 into two parts. $90 \%$ of the light is directed towards beam splitter 1 . $10 \%$ of the light is directed towards QPD 2. The light from fiber collimator 1 has propagated via beam splitter 1 and retroreflector 1 and 2 to beam splitter 2. At this beam splitter another combination of the light beams from fiber collimator 1 and 2 happens and the science interferometer is formed. Both beams travel through 2 lenses and, an aperture towards the quadrant photodiode 2 (QPD2), where the beat note between both beams is detected. Both lenses form an imaging system, which images the input aperture of the OBA onto the photodiode. In this way diffraction rings of the input aperture on the photodiode are avoided and possible beam pointing movements of the RX beam on the photodiode are suppressed.

Both interferometers of the OBA use quadrant photodiodes. These photodiodes can be used to perform the optical path length measurements, but also differential wave-front sensing. Differential wave-front sensing enables the PoC test to directly distinguish an alignment change of the optical beams from an optical path length change. Moreover, the use of a QPD in each interferometer allows to distinguish a beam pointing change of the fiber collimators from a beam pointing change of the retroreflectors and the ATT2 OGSE.

The optical path length change between the retroreflectors is derived from the relative phase of the beat signals between the two laser beams (local oscillator beam and the TX/RX beam) superimposed on the photodiodes QPD1 (reference interferometer signal) and QPD2 (science interferometer signal). The frequency of the beat signal on QPD1 corresponds to the frequency shift (heterodyne frequency) introduced between the local oscillator and the TX beam, which is $2 \mathrm{MHz}$ in the realised design. The frequency of the beat signal on QPD2 is the heterodyne frequency plus or minus the Doppler shift induced by the movement of the target (in the test setup the simulated movement of the retroreflectors in addition to any optical path length changes in the ATT2 OGSE and the OBA itself). Given the relative velocity between the spacecraft in orbit, a frequency variation of up to $\pm 188 \mathrm{kHz}$ is expected around the $2 \mathrm{MHz}$ beat note frequency. 
The beam propagation from RR1 to RR2 is placed above the OBA to avoid an additional aperture in the OBA and to reduce its complexity while at the same time being able to work with relatively small retroreflectors of $125 \mathrm{~mm}$ diameter. The retroreflector RR1 is rigidly installed on the optical table and retroreflector RR2, representing the remote $\mathrm{S} / \mathrm{C}$, is installed on a linear translation stage to mimic the relative motion between the $\mathrm{S} / \mathrm{C}$. The distance between the retroreflectors isl be kept as short as possible to minimize the optical pathlength noise in the setup. The attenuation of the laser intensity (attenuation factor between $10^{\wedge} 10$ and $10^{\wedge} 11$ ) - caused by the propagation between the S/C -is achieved in ATT2 through a high splitting ratio mirror and neutral density filters along the return path of the measurement beam.

The $\mu$ optical path length noise between the two retroreflectors is -at least in the low frequency region - expected to be above the required level of $2 \mathrm{~nm} / \operatorname{sqrt}(\mathrm{Hz}) \times \mathrm{NSF}(\mathrm{f})$, since the retroreflectors are approximately $1 \mathrm{~m}$ apart, the POCD is located in air and the setup is mounted on a steel optical table. Thus, an auxiliary interferometer (not shown in Figure 1) is used to measure the optical path length changes between the retroreflectors. The auxiliary interferometer cannot measure the exact optical path length as its measurement beam cannot be overlapped with that one of the OBA and does not run through ATT2. Nevertheless, for low frequencies $(<<1 \mathrm{~Hz})$ the optical path length change difference between both beam paths is assumed to be smaller than the required optical path length measurement noise.

The temperatures of the POCD are measured at certain points to identify possible correlations between temperature and optical path length trends measured by the OBA and the auxiliary interferometer. The POCD is installed in air on an optical table to improve its mechanical stability and suppress the influence of environmental vibrations (damped by the pneumatic isolators of the optical table place on a floor endowed with an anti-seismic block) onto the POCD. The optical table does have an enclosure to suppress thermal and air fluctuations at the POCD and additionally turbulence is reduced using an additional small enclosure.

All measurements have been done with the environment light switched on. No long measurement in dark has been done, the effect is considered negligible due to the incoherent nature of the light.

\section{Optical Bench Assembly Design (OBA) and Analysis}

The opto-mechanical design of the OBA was started using a sketch similar to the one presented in Figure 1. This level of detail is sufficient to review the implementation of the functional requirements and to review certain performance requirements, e.g. the optical link budget. This sketch was afterwards transferred to an optical layout in OpticStudio and the opto-mechanical properties of all optical components were integrated. The optical components were pre-aligned and, in a following step, the interferometer signal on both photo receivers were optimized. This optical layout was then used for the development of the mechanical design. In parallel, first investigations on the straylight with only the optics were performed. Afterwards, the iterative process between optical and mechanical design started. The final opto-mechanical design of the OBA is presented in Figure 2. The cross section shows the optic components, the housing and the first order optical beam paths of the OBA. The straylight analysis of the final OBA design took into account surface scattering and the reflections at the optical surfaces. The analysis itself concentrated on straylight suppression in comparison to the influence of beam pointing noise on measurement performance due to the fact that the assumed satellite platform of NGGM has a significantly higher pointing performance compared to the pointing and pointing noise of the LRI on GRACE-FO, namely in the order of $\mathrm{mrad}$ and $0.3 \mathrm{mrad} / \sqrt{ } \mathrm{Hz} \times \mathrm{NSF}(\mathrm{f})$.

For the surface scattering two contributions need to be taken into account: the first is the surface roughness and the second one is contamination by dust and particles. For the first one, the parameters for the $\mathrm{ABg}$ model can be directly calculated from the surface roughness following the derivation given in [5]. This is valid as long as the rms surface roughness is in the range of $\lambda / 100$ of the operating wavelength. The Total Integrated Scatter (TIS) can also be directly calculated from the surface roughness. The surface quality of the optics planned to be used is specified to be $5 / 1 \times 0.04$ (ISO 10110) and measurements of the roughness of the optics resulted in $\sigma=12 \mathrm{~nm} \mathrm{rms}$. This corresponds to $\lambda / 85$, which is close enough to the required roughness. For this roughness the TIS is about $1 \%$. The parameters $\mathrm{B}$ and $\mathrm{g}$ are fixed to 0.001 and 1.5 , respectively, see [5], and A is calculated to 0.001087 .

Ref. [6] provides a reworked and extended analysis for the scattering by dust/particle contamination. There, the forward and back scattering Bidirectional Reflectance Distribution Functions (BRDF) are separated which allows to apply the 
results to transmission optics as well. In addition, the range of the BRDFs extends down to lower angles $\left(1 \times 10^{-4}\right.$ degree or $1.7 \mu \mathrm{rad})$. The BRDF for different cleanliness levels is calculated. The investigations is done for a wavelength of 633.8 $\mathrm{nm}$, but a wavelength scaling of the BDRFs is possible. The procedure to use these BDRFs for the straylight analysis is as follows. ABG models for BRDFs can be directly utilized by Optics Studio, but an ABG model cannot accurately describe a BDRF and therefore BRDFs are reproduced as sums of ABG functions. Finally, a wavelength scaling of the parameters of the ABG function is performed according to [7]. This results in a BRDF at the correct wavelength, cleanliness class and for the forward and backward direction, which can be applied to the surfaces of the OBA optics. The particle contamination of the OBA is modelled using the information provided in ECSS-Q-ST-70-01. Finally, for the scattering of surface contamination, a cleanliness level of 300 according to MIL-STD-1246 is supposed. This surface contamination level corresponds to visibly clean after inspection by bare eye and can be seen as worst-case scenario.

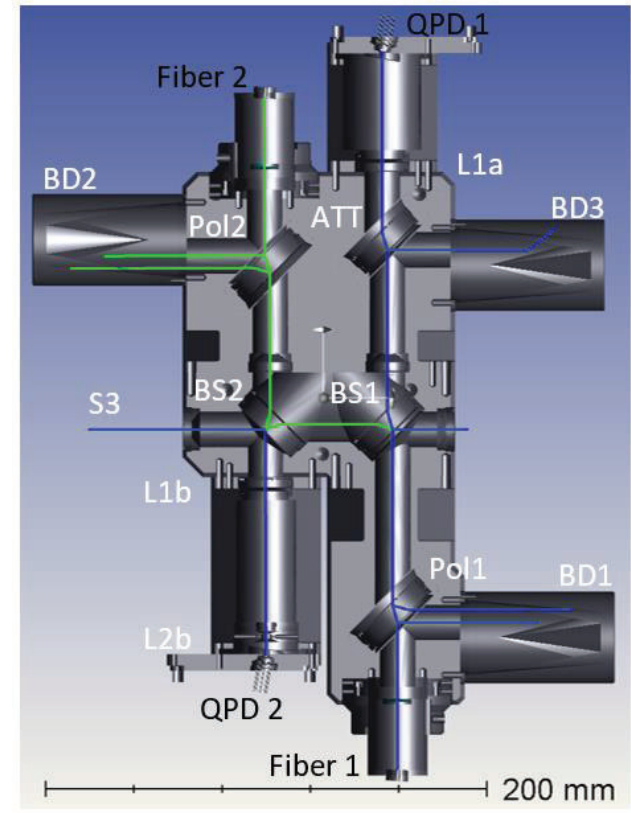

Figure 2 Cross section of the OBA in OpticStudio, which show the optic components, the housing and the first order optical beam paths.

The TIS is compared for different contributions -in Figure 3-to evaluate the influence of scattering by surface roughness and surface contamination. The TIS is the amount of energy distributed from the specular direction. The red bar in Figure 3 indicates $10 \mathrm{mrad}$. Below this angle the scattering contribution to the straylight is smaller than the specular reflections represented by the curves of the AR coatings. The heterodyne signal vanishes for angles larger than 1 mrad between beams (lesson learnt from LRI OBA). Therefore, the scattering from surface roughness and surface contamination of the optical components has not to be taken into account for the straylight analysis as they only have a minor contribution to the total amount of straylight.

Scattering analysis in OpticStudio included scattering at OBA structure elements. A review of the scattering at the OBA structure elements revealed that direct reflection at the OBA structure elements will give a worst case estimation of the possible straylight in comparison to scattering. Thus, scattering at OBA structure elements was implemented by direct reflections.

The most important features of the OBA design to achieve the required straylight suppresion of $1 \times 10^{15}$ to $1 \times 10^{17}$ with regard to the $500 \mathrm{~mW}$ output power of the TX beam will be described in the following. It is important to note in this context that OpticStudio has a lower limit of $1 \times 10^{-17}$ for the optical power dynamic range, meaning the analysis is operation at the limit of the software capabilities. The beam splitters BS1 and BS2 in Figure 2 have a wedge of 1 mrad circa between the front and back surface. This wedge has the function that spurious reflections of the LO and TX beam at the AR coating side lead to significantly reduced beat note amplitude compared to the proper LO and TX beam. As stated above the heterodyne signal level is strongly depending on the angle between the laser signals. The $-3 \mathrm{~dB}$ acceptance cone of the 
associated interferometer signals is approx. $100 \mu \mathrm{rad}$ and above $1 \mathrm{mrad}$ the signal is suppressed by more than 2 orders of magnitude. The beam splitter ATT is identical to BS1 but for this beam splitter the wedge is not a required characteristic. The beam splitter ATT serves the function that any straylight, which is generated after it (between ATT and the photo receiver QPD1) is suppressed by a factor of approx. 100, when it passes the beam splitter ATT again (into the direction to beam splitter BS1).

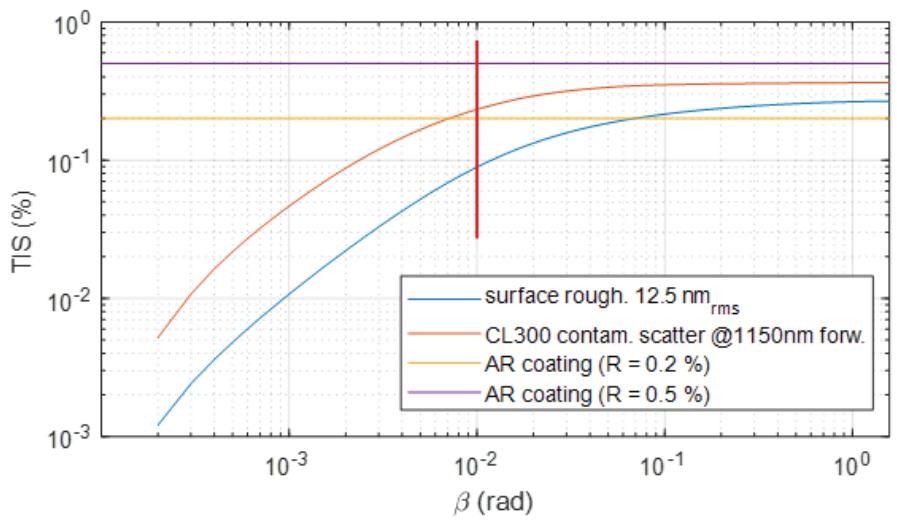

Figure 3 Total integrated scatter for surface roughness and surface contamination in comparison to AR coatings.

The glass windows of the photodiodes have been removed from the beginning in the optical design to avoid any multipath reflections. The AR coating of the COTS photodiodes is optimized for $1310 \mathrm{~nm}$ and not for $1064 \mathrm{~nm}$. The removal of the glass window will avoid straylight from the window itself and to avoid possible cavity effects between the glass window and active area of the photodiode. The active area itself reflects a significant amount of the light back towards the setup. The active area is usually AR coated but this coating serves the purpose to transfer more light into the photodiode chip. The reflection of the light, which is not absorbed by the photodiode chip, occurs at the back of the photodiode chip, where the chip is usually metallized. Thus, the photodiodes have been angled by $10^{\circ} \mathrm{wrt}$. the impinging beams to suppress spurious interference with the LO and TX beams. Additional apertures have been installed in front of the photodiodes to absorb the back reflected light from the photodiodes.

The combination of the measures above resulted in an OBA design that is expected to achieve the required straylight suppression of at least $1 \times 10^{15}$. However, due to the various assumptions and model limitations at this extreme straylight suppression requirement, the experimental verification is absolutely essential to confirm the achievable performance on real hardware, which is described below.

Figure 4 shows the assembled and pre-tested OBA in the cleanroom at SpaceTech in Immenstaad (DE). The black-coated parts are parts, which have a commercial build-standard or have not been manufactured for space operation. All the other uncoated metal parts follow the design rules, which have also been used during the qualification of the Laser Ranging Instrument for GRACE-FO. 


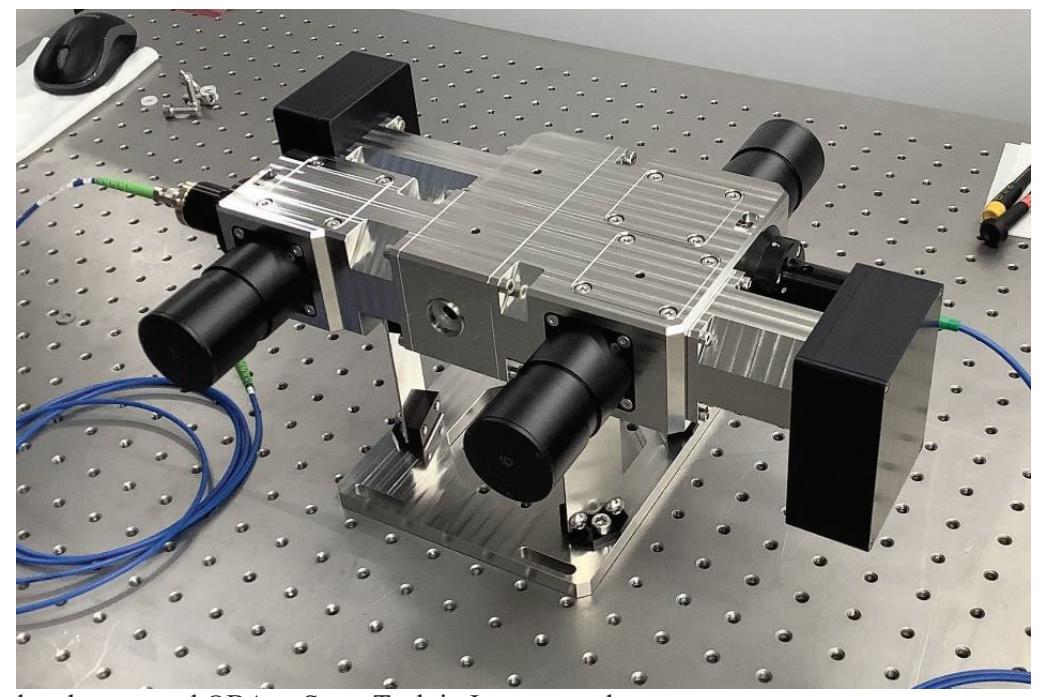

Figure 4 Assembled and pre-tested OBA at SpaceTech in Immenstaad.

\section{Optical Round-trip GSE}

The initial schematic of the POCD setup with emphasis on the GSE is shown in Figure 5. The optical round-trip GSE has to simulate the most important characteristics of the round-trip between the two NGGM spacecraft. These are the optical connection of the output and input aperture of the OBA with a beam co-alignment error well below $100 \mu \mathrm{rad}$ and the attenuation of the optical beam corresponding to the in-orbit variable distance between the satellites. The beam propagation (change of beam diameter and wave front) has not to be covered with the optical round-trip GSE. The arising difference in the heterodyne efficiency between the RX and LO signal in the science interferometer is compensated by adapting the signal power of the RX signal correspondingly. Additionally, an auxiliary interferometer (AUX) is installed in the POCD, which measures the optical path length noise between the two retro-reflectors, introduced by the optical round-trip (being in air) and the thermal noise. Its measurement is used in the data analysis to suppress these noise contributions.

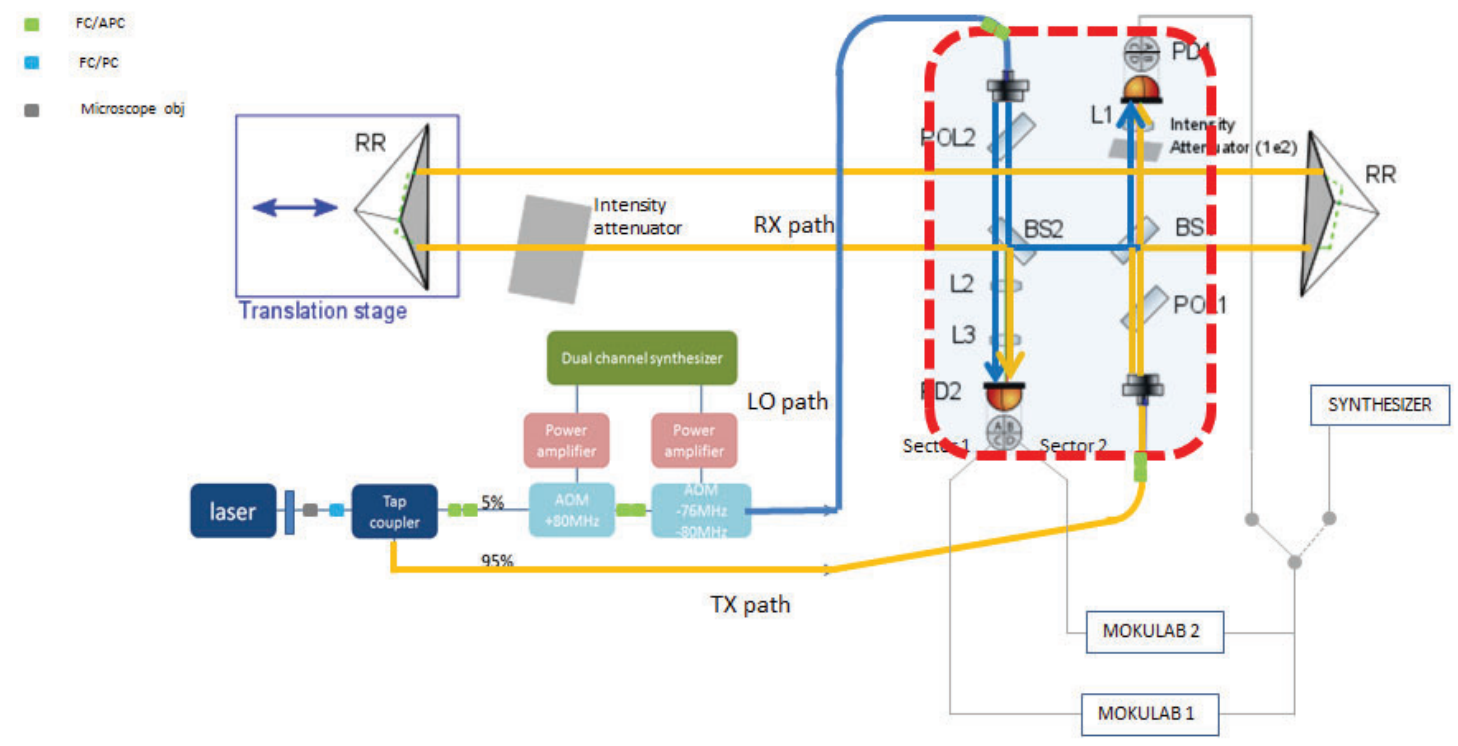

Figure 5 Initial schematic of the POCD showing the GSE. 
Two commercial of the shelf (COTS) corner cube retroreflectors from Edmund Optics have been selected to connect the output and input aperture of the OBA. The selected corner cube retroreflectors have a significantly larger aperture in comparison to the possible FM design. The aperture is larger to accommodate a second COTS auxiliary interferometer (Keysight 5530) within the aperture of the retroreflector and measure optical path length noise of the round-trip independently. Using this interferometer setup, the optical path length noise of the round-trip measured by the OBA can be improved by subtracting the optical path length noise measured by the auxiliary interferometer. The latter one only measures the round-trip between the retro-reflectors and will not pass through the OBA and the attenuation optics. Thus, the correction via the auxiliary interferometer will not be perfect. Since the retroreflectors are large in comparison to the FM retroreflectors, the beam co-alignment error of the retroreflectors is relatively large, too. A set of two tunable high stability tip-tilt stages with HR mirrors as part of the attenuation GSE have being used to align the received beam to the local oscillator beam in the OBA.

The intensity attenuator is based on standard neutral density filters from Thorlabs. The overall attenuation of the intensity attenuator can be changed by changing the quantity of the implemented neutral density filters and their individual attenuation. The individual attenuation of the neutral density filters has been measured to be able to set the attenuation very accurately, and the straylight level of the filters have been checked as well.

A linear translation stage allows the setup to simulate physically relative velocities of the corner cube retroreflectors up to $0.06 \mathrm{~m} / \mathrm{s}$; velocities up to $0.1 \mathrm{~m} / \mathrm{s}$ can be simulated with an additional synthesizer whose purpose is to simulates Doppler shift. The test setup on the optical table is shown in Figure 6.

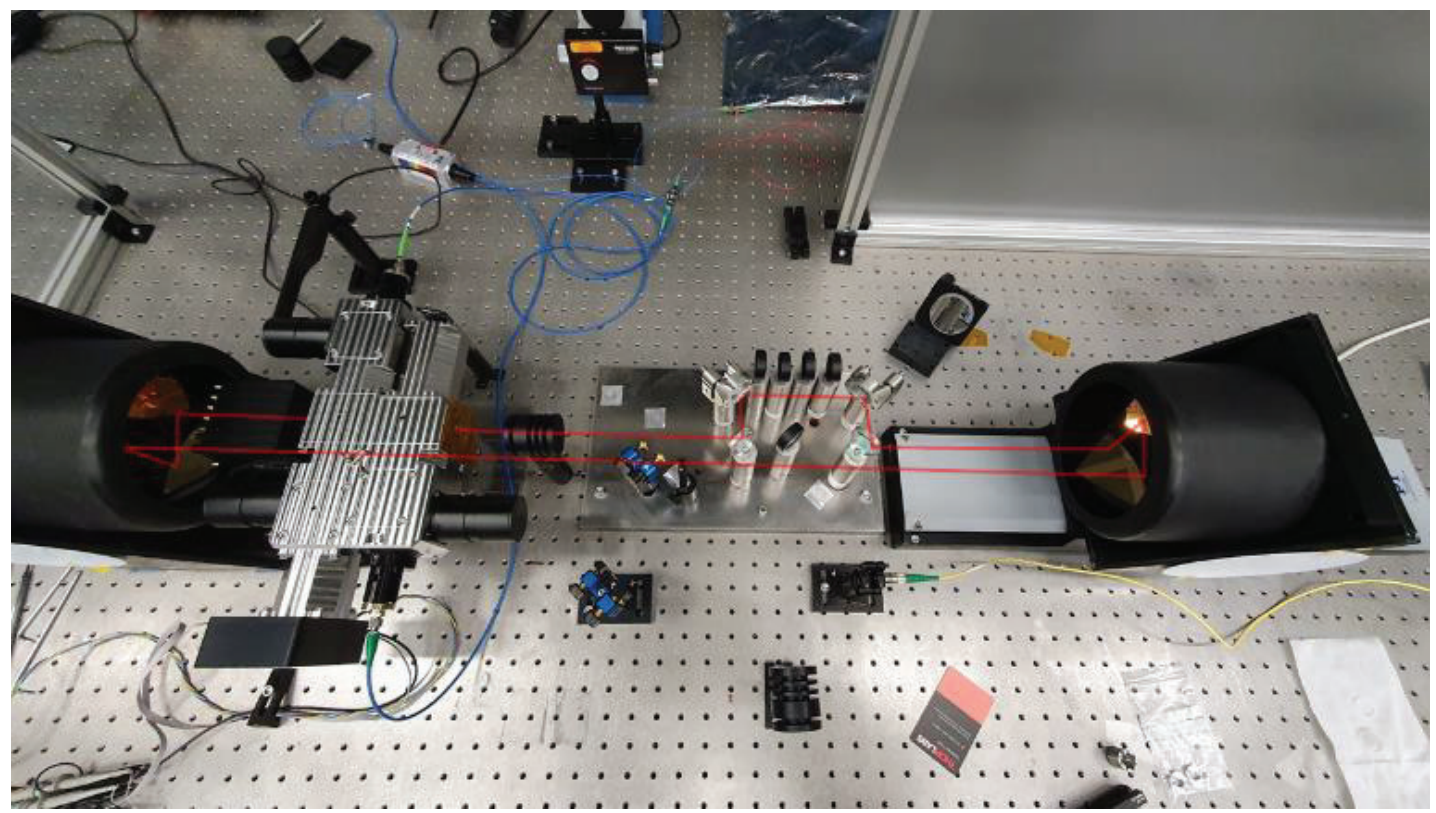

Figure 6. POCD test setup on optical table at ThalesAlenia Space Turin (IT). In red, the optical path is sketched.

\section{Laser Source GSE}

The laser source GSE has to reproduce the important characteristics of the FM laser system. Later on, this one will likely be based on a NPRO, which has an output power of approx. $25 \mathrm{~mW}$. The light from this laser will be split into the LO and TX signal, whereby the TX signal is amplified with a fiber power amplifier to the required $500 \mathrm{~mW}$. The LO and TX signals will be shifted in frequency with respect to each other using AOMs.

In the PoCD setups the primary laser source is a Coherent (formerly Innolight) Prometheus 20 laser with iodine stabilization used to provide a frequency stabilized laser source with a wavelength of $1064 \mathrm{~nm}$ and an output power of up to $1 \mathrm{~W}$. The frequency stability of the laser is at a level of $40 \mathrm{~Hz} / \sqrt{ }(\mathrm{Hz})$ at $1 \mathrm{~Hz}$ and is increasing with a $1 / \mathrm{f}$ slope for 
frequency below $30 \mathrm{mHz}$. This frequency stability is sufficient for the POCD since it will not limit the optical path length noise for the implemented arm length mismatch of approx. $2 \mathrm{~m}$. The Prometheus laser has a noise eater to suppress the relaxation oscillation of the NPRO laser from $300 \mathrm{kHz}$ to $700 \mathrm{kHz}$. For the POCD test campaign the noise eater is always switched on.

The primary laser source optical power is split in power using a tap coupler from Gooch \& Housego. This tap coupler provides $5 \%$ of the light to the Local Oscillator (LO) beam collimator and $95 \%$ of the light to the transmit (TX) beam collimator. The TX signal becomes the receive (RX) signal after the round-trip and the associated attenuation.

The local oscillator signal is shifted in frequency by $2 \mathrm{MHz}$ using two acousto-optic modulators (AOMs) in series. Fiber coupled AOMs from AA Opto-Electronic have been used for this purpose. They can shift the frequency from $78 \mathrm{MHz}$ to $82 \mathrm{MHz}$ and are set to a difference frequency of $2 \mathrm{MHz}$. The AOMs are located within the LO signal path in the initial setup, because this signal path does only transport a small percentage of the available $500 \mathrm{~mW}$ optical power from the laser source and the influence of the insertion loss of the AOMs (approx. $3 \mathrm{~dB}$ per piece) is therefore smaller than in the high power beam. The AOMs are driven by a standard dual channel signal generator and two independent power amplifiers.

\section{Read-out chain GSE}

The read-out chain GSE is presented in Figure 7. Let's start with the photo receiver. The photo receiver in the reference and in the science interferometer are almost identical. Both photo receivers use the quadrant photodiode FCI-InGaAsQ1000 from OSI opto-electronic, which were also used for the LRI on GRACE-FO. The most important reasons, why these photodiodes have been selected, are the following. Firstly, the quantum efficiency of InGaAs is higher than that of Silicon. Secondly, the capacitance of the photodiode is sufficient to achieve a bandwidth in the MHz range with a tens of $\mathrm{kOhm}$ trans-impedance gain. Third, the active area of the photodiode is compatible with the imaging optics characteristics.

The following trans-impedance and amplification stage components have been selected from COTS components to achieve a very good signal-to-noise ratio within the science interferometer. This was done because the goal of the POCD is to identify possible straylight sources within the OBA and thus the SNR in the science interferometer was designed to be much better than required to not limit the straylight detection capabilities by the read-out noise when using only flightcompatible components. The overall amplification of the detector chain is different in the reference and science interferometer due to available signal amplitude from the LO and RX/TX beams.

Due to the fact that no phasemeter with eight channels is available in the actual POCD, the four channels of each photo receiver are summed and provided to the available MokuLab Phasemeter.

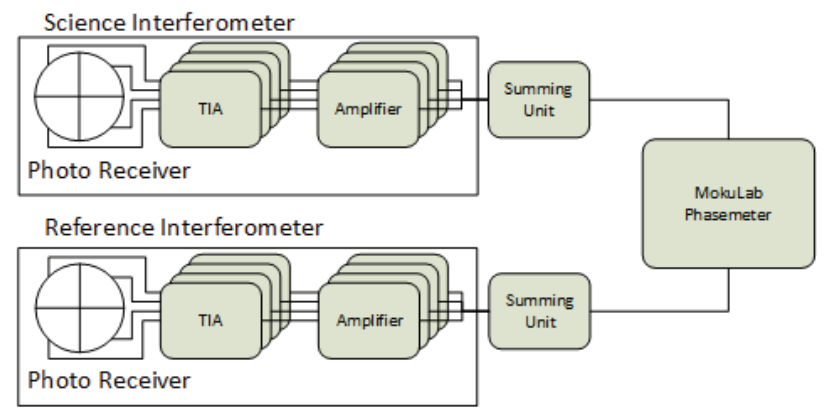

Figure 7 Schematic of the read-out GSE.

\section{PRELIMINARY TEST RESULTS}

A spurious beat note at $2 \mathrm{MHz}$ on the science signal was observed at the beginning of the test campaign. The spurious signal was observed in the spectrum of the science signal and influenced the phase measurement of the science signal to a level above the optical phase noise requirement for a RX signal level, which was also above the required sub pico Watt level. The source for this beat note at $2 \mathrm{MHz}$ was identified to be related to two different sources: internal cross-talk between the two channels of the synthesizer (the spurious noise almost halved substituting the dual channel synthesizer 
with two synchronized separate synthesizers) and the optical cross-talk within the fiber coupled AOMs itself. The crosstalk of adjacent diffraction orders within each AOM is specified to be $1 \times 10^{-5}$. When the LO light passes two AOMs a beat note at $2 \mathrm{MHz}$ is generated, which has a relative amplitude of $1 \times 10^{-10}$. This is depicted in Figure 8 . The light with frequency $v_{0}$ enters the first $A O M$ and is shifted in frequency by $+f_{1}$. Due to the diffraction order cross-talk a portion $R$ of $v_{0}$ enters the fiber towards the next AOM, too, where $\mathrm{R}$ is the cross-talk coefficient. In the second AOM both signals are shifted in frequency by $-\mathrm{f}_{2}$ but additionally a portion $\mathrm{R}$ of the zero diffraction order signals enter the fiber. The wanted signal is the signal which has the frequency $\left(v_{0}+f_{1}-f_{2}\right)$ but unfortunately a beat note between the signal $R^{2} v_{0}$ and signal $\left(v_{0}+f_{1}-f_{2}\right)$ is generated, too. The straylight signal is not a problem for the reference interferometer because its relative amplitude wrt. the reference signal is small due to the $\mathrm{R}^{2}$. However, in the science interferometer, because the beam that is travelling from the OBA to RR1, RR2 and the attenuation GSE is attenuated by $1 \times 10^{11}$, the $\mathrm{R}^{2}$ leads to a straylight signal order above the required level of $1 \times 10^{-15}$. The two signals at the other frequencies are filtered by the bandwidth of the photodetectors.

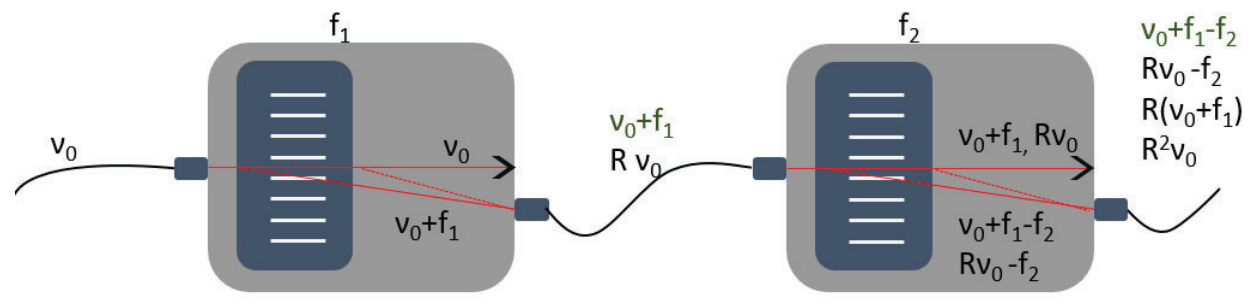

Figure 8 Optical cross-talk of diffraction orders in the AOMs of the laser source GSE.

Two solutions have been identified to overcome this problem. The first is to develop a free-space or fiber coupled AOM modulation bench similar to LISA Pathfinder one [8] in which the AOM is in the LO path and on in the TX path. The other one is to move the AOMs from the LO signal path to the TX signal path. The first solution overcomes the observed straylight problem because the diffraction order cross talk is distributed to the LO and the RX signal and the interference occurs between the signals $\left(v_{0}+f_{1}\right)$ and $\left(v_{0}+f_{2}\right)$. The straylight signal is at DC and is not seen by the phase measurement system. The first solution was discarded due to time constraints; the lead time for the additional hardware and the development time for the modulation were not compatible with the schedule. The second solution overcomes the observed straylight problem because the stray light signal is attenuated in the same way as the TX signal. Thus, the straylight becomes uncritical also for the science interferometer. The second solution was selected for the continuation of the test campaign. The drawback of this solution is that the TX power is reduced from approx. $500 \mathrm{~mW}$ down to $125 \mathrm{~mW}$ : the attenuation within the round-trip was reduced in the same manner and the influence of the reduced TX power on the straylight level within the OBA was reviewed. The assumption is that the straylight is reduced by the same factor as the TX power, which ultimately limits the representability of the measurements.

The POCD test campaign focussed on the optical path length noise with the lowest optical power level expected in orbit, concerning the RX beam. This is due to the fact that the straylight, which is generated within the OBA, will have the highest influence at the lowest possible RX power level. For the data analysis the LTPDA (LISA Technology Package Data Analysis) Toolbox [4] was used, which is a MATLAB toolbox that uses an object-oriented approach to data analysis and provides accountable and reproducible data analysis.

Figure 9 shows an exemplary time-series of the phase measurement of the reference (QPD1) and the science interferometer (QPD2) measured by the MokuLab Phasemeter, and the time-series of the auxiliary interferometer (AUX). The time-series of the round-trip phase measurement is the difference of the reference and science interferometer. The phase noise of the reference and science interferometer does contain the common-mode noise of the laser source GSE up to the combination beam splitters. This common mode noise is reduced significantly, when the reference phase is subtracted from the science phase. The round-trip shows only a slow drift but not the fast variation of the phase any more. The round-trip and the AUX phase seem to be influenced by the same phase drift for longer time periods, indicating that the two independent interferometers measure consistent data. 


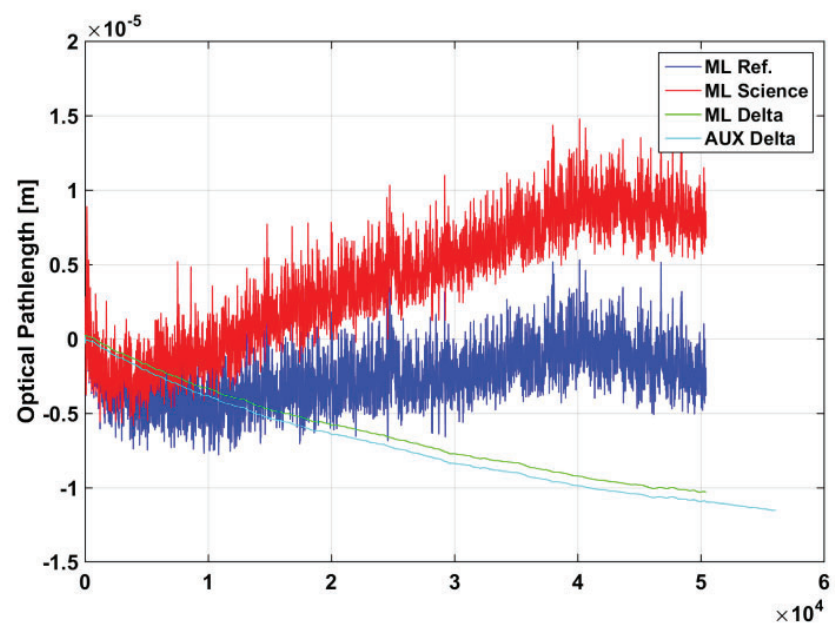

Figure 9 Time-series of the phase measurement of the reference (ML Ref.), the science (ML Science) and the AUX (AUX Delta) interferometer. The round-trip phase noise (ML Delta) is the difference between the reference and the science interferometer.

Figure 10 shows the optical pathlength noise of the time-series presented in Figure 9. For the generation of the spectra the MokuLab data and the auxiliary data are resampled to the same sampling frequency and the spectra were calculated for the time period starting at 20.000 seconds and ending at 40.000 seconds. The raw photoreceiver data of reference and science detector (QPD 1 and QPD2, read and green (green is barely visible)) show almost on the same noise level. They are starting at $1 \mathrm{~Hz}$ at around $5 \mathrm{~nm} / \sqrt{ }(\mathrm{Hz})$ and are increasing towards lower frequencies up to $10 \mu \mathrm{m} / \sqrt{ }(\mathrm{Hz})$ at $10 \mathrm{mHz}$.

The round-trip optical pathlength noise (difference between reference and science interferometer) is significantly better than the raw phase of the interferometers. For frequencies above $50 \mathrm{mHz}$ the optical pathlength noise is below the required level of $2 \mathrm{~nm} / \sqrt{ }(\mathrm{Hz})$ and for lower frequencies the optical pathlength noise is increasing with a 1/f slope. The optical pathlength noise of the auxiliary interferometer is only for frequencies above $800 \mathrm{mHz}$ better than the required level of 2 $\mathrm{nm} / \sqrt{ }(\mathrm{Hz})$. For frequencies below $3 \mathrm{mHz}$ the auxiliary interferometer phase noise is almost identical with the round-trip phase noise and thus for this frequency range the auxiliary interferometer can be used for the correction of the round-trip phase noise. A transfer function between the auxiliary interferometer phase and the round-trip noise was calculated to verify this assumption. The information from the transfer function was used to filter the auxiliary interferometer phase and subtract it from the round-trip phase. The result is shown in the corrected round-trip phase noise curve: for frequencies below $1 \mathrm{mHz}$ the round-trip phase noise is improved by this process. 


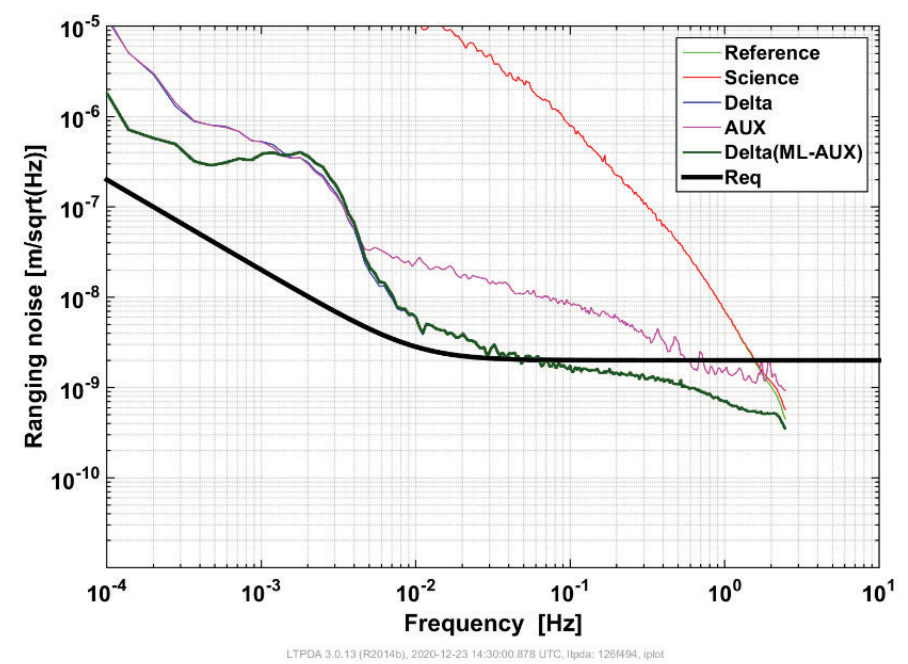

Figure 10 Spectra of the phase measurements of the reference, the science and the AUX interferometer. The round-trip phase noise (Delta) is the difference between the reference and the science interferometer. The correction of this round-trip measurement with the measured AUX interferometer phase is (Delta (ML-AUX))

Figure 11 shows the measured time-series of the temperature sensors, which are installed at different location of the POCD, during the measurement presented before. All time-series show a slow drift over a temperature range of $0.3^{\circ} \mathrm{C}$ over the time period of approx. 55000 seconds. The temperature sensor on the invar bench of the round-trip GSE (Bench No.2) shows a small temperature step at around 15000 seconds, which is assumed to be a measurement artefact. Beside this, all temperature sensors seem to show the same long and short term behavior.

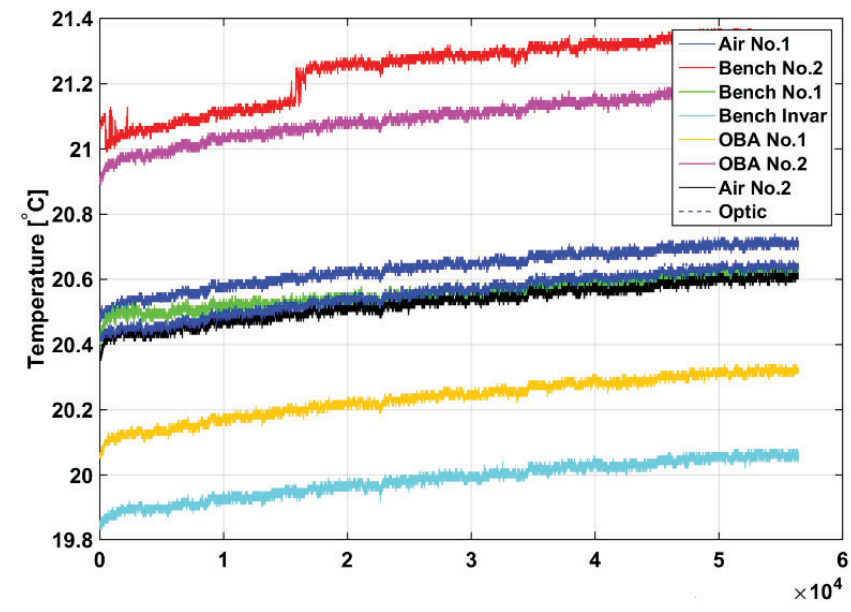

Figure 11 Time-series of the measured temperatures at POCD different locations.

Figure 12 shows the temperature noise of the time-series presented in Figure 11. The spectra were calculated for the time period starting at 20000 seconds and ending at 40000 seconds. The spectra show that the temperature sensors in Air, on the Invar bench and on the OBA have a similar temperature noise spectrum. Additionally the difference between two nearby sensors were taken to verify that the temperature read-out is not limited by the temperature measurement system. These are the Delta curves. The read-out of the air and OBA sensors is not limited by the temperature measurement system 
for frequencies below $100 \mathrm{mHz}$. For the temperature sensor on the Invar bench this is not the case. It is assumed that this is introduced by a damage of the temperature sensor "Bench No.2", which needs to be repaired.

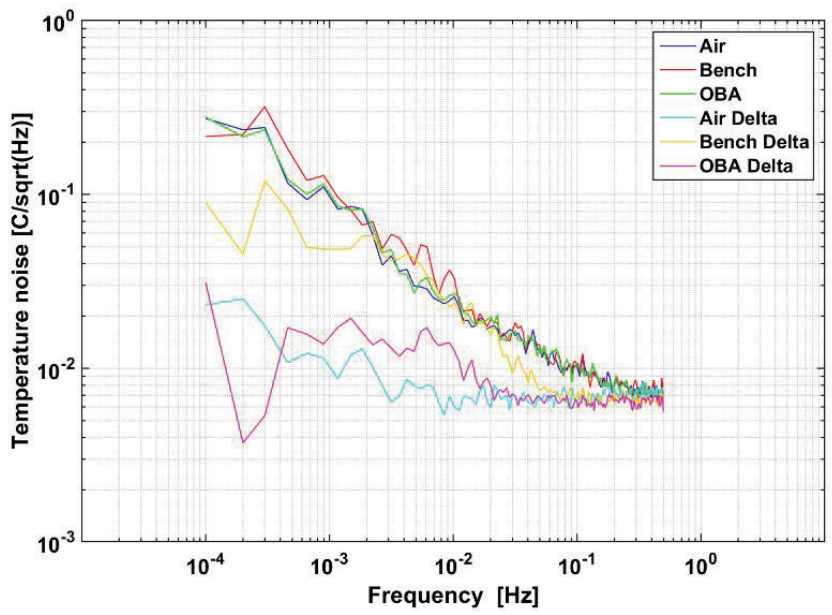

Figure 12 Spectra of the measured temperatures at the POCD.

\section{CONCLUSION}

In this activity the optical design and the manufacturing, assembly and testing of an OBA for the NGGM off-axis retroreflector interferometer candidate concept was performed to verify the feasibility to achieve the challenging straylight suppression of at least $1 \times 10^{15}$. The optical analysis of the OBA for the off-axis retroreflector interferometer configuration for NGGM predicts a sufficiently low straylight level to achieve the required optical path length noise within the associated optical link budget. The contamination analysis revealed that dust and particles are uncritical in comparison to the influence of AR coating on the straylight level. The cleanliness of optic needs to be visibly clean, which is well above the assumed cleanliness of the optics inside the OBA.

The preliminary POCD test results show an optical path length noise, which is below the requirement above a frequency of $50 \mathrm{mHz}$, indicating that straylight is indeed highly suppressed. The source for the optical path length noise below $50 \mathrm{mHz}$ will be further investigated. The actual assessment is that the non-compliancy root cause is the test setup itself, more precisely the unstable temperature and the air fluctuations in the test setup.

A straylight source within the laser modulation bench has been identified and the level of straylight was successfully suppressed by order of magnitudes by changing the frequency shifters from the LO beam to the TX beam.

Further investigations on this proof-of-concept demonstrator are required to improve the level of maturity, both in terms of setup stability and TX power level, and to verify that the optical-path length noise and the low-level of straylight are achieved under all relevant environmental conditions. These investigations are planned currently.

Although more investigations are needed to increase the confidence in the design and to meet the requirements over the full science measurement band, the preliminary test results indicate that it may indeed be possible to reach the required straylight suppression with an optical bench design as described and tested. In addition, further measures have been identified in the course of this activity that may be implemented if the results of further investigations do not show sufficient performance. Among these, the authors have identified further apertures and polarization separation to further suppress straylight on the OBA side. On the modulation bench side, straylight can be further suppressed by chopping the TX beam and thereby separating the measurements in the reference and science interferometer in time. 


\section{ACKNOWLEDGEMENT}

The activity "Proof-of-concept Test for the Off-Axis Retroreflector Interferometer Configuration for NGGM" was funded by ESA under contract number 4000126531/19/NL/IA.

\section{REFERENCES}

[1] K. Abich et al. "In-Orbit Performance of the GRACE Follow-on Laser Ranging Interferometer", Phys. Rev. Lett. 123, 031101, July 2019

[2] Nicklaus, K., Cesare, S., Massotti, L. et al. Laser metrology concept consolidation for NGGM. CEAS Space J 12, 313-330 (2020). https://doi.org/10.1007/s12567-020-00324-6

[3] R. Haagmans, et. al. "ESA's next-generation gravity mission concepts. Rend. Fis. Acc. Lincei (2020)". https://doi.org/10.1007/s12210-020-00875-0

[4] Hewitson, M., "LTPDA; a MATLABC toolbox for accountable and reproducible data analysis", https://www.elisascience.org/ltpda/index.htmlhttps://www.elisascience.org/ltpda/

[5] Pfisterer, Approximated scatter models for stray light analysis, Optics, 2011

[6] Dittman, Contamination scatter functions for stray-light analysis, SPIE Proc. 4774, 1992

[7] Lambda Research, Abg Lambda Wavelength scaling, Technical Note, 2018

[8] Interferometry at low frequencies: Optical Phase Measurement for LISA and LISA Pathfinder, PhD thesis, Vinzenz Wand, 2007

[9] Massotti L., Amata G.B., Anselmi A., Cesare S., Martimort P., Silvestrin P., "Next Generation Gravity Mission: status of the design and discussion on alternative drag compensation scenarios", Proc. of SPIE Remote Sensing 2020, Edinburgh, UK, September 2020, DOI: 10.1117/12.2573924 